\title{
The Effect of a Sequential Contraction Compression Device on a Hypoesthetic Diabetic Foot: A Case Report
}

\section{Rosenblum $\mathbf{J}^{1 *}$, Greenberg $\mathbf{N}^{2}$ and Weiss $\mathrm{S}^{2}$}

${ }^{1}$ Diabetic Foot Service, Department of Orthopedics, Shaarei Zedek Medical Center, Jerusalem, Israel

${ }^{2}$ Foot and Ankle Service, Department of Orthopedics, Shaarei Zedek Medical Center, Jerusalem, Israel

\begin{abstract}
This report discusses the use of a novel compression therapy in a patient with severe chronic hypoesthetic Diabetic Peripheral Neuropathy. After commencing use of the device, the patient who previously was insensate in his feet, and as a result had balance and ambulation issues had a significant resolution of his symptoms.
\end{abstract}

Keywords: Neuropathy; Diabetic complications; Compression therapy; Electric muscle stimulation

\section{Introduction}

Diabetic Peripheral Neuropathy (DPN) is a debilitating complication of Diabetes. There are three primary presentations to DPN: Hyperesthetic, Hypoesthetic and Mixed. Patients suffering from hypoesthetic DPN have a lack of sensation and oftentimes complications with proprioception. This develops into problems with ambulation and becoming prone to falls. In addition, hypoesthetic DPN is a leading cause in the development of Diabetic Foot Ulcerations in the forefoot, which has its own series of complications [1-3].

Until recently DPN was described as a metabolic disease. The argument as to its etiology centered around how exactly the hyperglycemia affected the nerve fibers [4,5]. Of late there has been new research that showed that at least a portion of the development of DPN was vascular or more specifically ischemic in nature. The current understanding is that with the development of fibrin cuffs around the capillaries that feed the nerves, there is a progression of microischemia leading to a slow death of the nerve fibers [6,7]. This has led to a paradigm shift in the outlook to and treatment of DPN [8].

SCCD works by sending a timed electric pulse into the muscles of the leg. The specific pattern of electric impulses causes a peristaltic series of contractions of the leg muscles that compress the veins in the leg. This causes a significant increase in venous outflow. On the release of the impulse there is a significant increase in arterial inflow. In a patient whose macrovasculature is unimpaired but has a damaged microvascular system, i.e., the capillaries to the nerves, the impulse speed can be increased leading to a hyper perfusion of the limb and a flooding of these capillaries. The result is an increased pressure causing a pressure induced expansion and destruction of the fibrin cuff and a reperfusion of the nerve. As a result the nerve fiber can repair and regenerate itself, and the symptoms can pass.

\section{Case Report}

M.B., a 74-year-old male, suffers from Type 2 Diabetes for more than 30 years. He is somewhat controlled $(\mathrm{HbAlC}<8.0)$. M.B. is a non -smoker. He is significantly obese $(\mathrm{BMI}>30)$. Over the course of the last 8 years M.B. has been complaining of worsening numbness and tingling that began in his toes and for the last three years has had total paresthesia of both of his feet, and currently also above the ankles. He has been forced to use a walker to aid ambulation because of balance issues and relates no less than one fall a month. He is unable to walk more than 50 meters because of "strange sensations" in his legs.
On examination M.B. had a negative Semmes-Weinstein test on all eight locations. He had a moderately negative proprioception test of the hallux. He had negative two-point discrimination. He had palpable and slightly bounding DP and PT pulses. His skin temperature was within normal limits. He had normal range of motion of the foot and ankle joints. His legs were moderately edematous with no pitting. SCCD therapy was initiated for a two-hour session. At the end of the session M.B. was asked to stand and promptly began to cry. When asked if he was in pain, he replied no, but was crying because he was able to feel the carpet, and this was a first for him in over three years. A follow up objective examination was immediately performed and no difference was found to before treatment.

SCCD therapy was continued for a month with a treatment regimen of two hours daily. At the end of the month M.B. was evaluated and there was a significant improvement to both the Semmes-Weinstein and two-point discrimination evaluation. The improvement was scattered both proximally and distally and followed no specific pattern. M.B. related to not needing the walker and used a cane instead but felt that he would soon want to discard that too. His leg edema was significantly decreased as well.

M.B. continues to use SCCD daily and his improvement is remarkable both in objective measures such as distance walked and sensation as well as subjective measures such as quality of life and satisfaction.

\section{Conclusion}

This case report highlights a novel use of SCCD. While a randomized controlled trial of SCCD is highly recommended this utilization of SCCD may represent a significant paradigm shift and new approach to the treatment of DPN. This is further highlighted by the sustained effect with continued use of the device.

*Corresponding author: Rosenblum J, Diabetic Foot Service, Department of Orthopedics, Shaarei Zedek Medical Center, Jerusalem, Israel, Tel: 97226666666 ; E-mail: diabfootman@gmail.com

Received May 25, 2016; Accepted June 16, 2016; Published June 26, 2016

Citation: Rosenblum J, Greenberg N, Weiss S (2016) The Effect of a Sequentia Contraction Compression Device on a Hypoesthetic Diabetic Foot: A Case Report. Med Rep Case Stud 1: 118. doi: 10.4172/2572-5130.1000118

Copyright: (c) 2016 Rosenblum J, et al. This is an open-access article distributed under the terms of the Creative Commons Attribution License, which permits unrestricted use, distribution, and reproduction in any medium, provided the original author and source are credited. 
Citation: Rosenblum J, Greenberg N, Weiss S (2016) The Effect of a Sequential Contraction Compression Device on a Hypoesthetic Diabetic Foot: A Case Report. Med Rep Case Stud 1: 118. doi: 10.4172/2572-5130.1000118

Page 2 of 2

\section{References}

1. Van Acker K, Bouhassira D, De Bacquer D, Weiss S, Matthys K, et al. (2009) Prevalence and impact on quality of life of peripheral neuropathy with or without neuropathic pain in type 1 and type 2 diabetic patients attending hospital outpatients clinics. Diabetes Metab 35: 206-213.

2. O'Connor AB (2009) Neuropathic pain: quality-of-life impact, costs and cost effectiveness of therapy. Pharmacoeconomics 27: 95-112.

3. Callaghan BC, Cheng HT, Stables CL, Smith AL, Feldman EL (2012) Diabetic neuropathy: clinical manifestations and current treatments. Lancet Neurology 11: $521-534$

4. Anonyms (1993) The Diabetes Control and Complications Research Trial Group. The effect of intensive treatment of diabetes on the development and progression of long-term complications in insulin-dependent diabetes mellitus. N Engl J Med 329: 977-986.

5. Anonyms (1998) Intensive blood-glucose control with sulphonylureas or insulin compared with conventional treatment and risk of complications in patients with type 2 diabetes (UKPDS 33). Lancet 352: 837-853.

6. Krishnan AV, Lin CS, Park SB, Kiernan MC (2009) Axonal ion channels from bench to bedside: a translational neuroscience perspective. Prog Neurobiol 89 288-313.

7. Fernyhough $P$ (2015) Mitochondrial dysfunction in diabetic neuropathy: a series of unfortunate metabolic events. Curr Diab Rep 15: 89.

8. Greig M, Tesfaye S, Selvarajah D, Wilkinson ID (2014) Insights into the pathogenesis and treatment of painful diabetic neuropathy. Handb Clin Neuro 126: $559-578$. 